Journal of Animal and Veterinary Advances 11 (14): 2459-2464, 2012

ISSN: $1680-5593$

(C) Medwell Journals, 2012

\title{
The Complete Genome of an Epidemic Strain of the Classical Swine Fever Virus Identified in China During 2010-2011
}

\author{
${ }^{1,}{ }^{2}$ Ruosong Yang, ${ }^{3}$ Jinqing Jiang, ${ }^{4}$ Peiyu Gao, ${ }^{3}$ Xingyou Liu and ${ }^{3}$ Jinyou Ma \\ ${ }^{1}$ College of Veterinary Medicine, Northwest A\&F University, 712100 Yangling, China \\ ${ }^{2}$ Zhangjiakou City Animal Disease Prevention and Control Center, 075000 Hebei, China \\ ${ }^{3}$ College of Animal Science, Henan Institute of Science and Technology, 453003 Xinxiang, China \\ ${ }^{4}$ Zhangjiakou City Aquaculture Technology Extension Station, 075000 Hebei, China
}

\begin{abstract}
The aim of this study was to identify the Classical Swine Fever Virus (CSFV) strain in isolates collected from 14 provinces in China between 2010 and 2011 by analyzing the sequences of the $E 0$ genes which are the major genes in CSFV. After collecting samples from pigs that have the clinical symptoms of a CSFV infection, researchers isolated viral RNA and obtained the $E 0$ gene by Reverse Transcription-Polymerase Chain Reaction (RT-PCR). Researchers analyzed the sequences of the $E O$ gene to determine the CSFV strain that caused the epidemic in China during 2010-2011. Finally, the complete genome of the epidemic strain was determined by RT-PCR analysis. The epidemic CSFV strain which was named HEBZ/CA/2010 was isolated from clinical samples obtained from the Hebei province of China and is 12296 Nucleotides (nt) in length. It is composed of a 372-nt-long 5'-terminal Untranslated Region (UTR), an 11697-nt Open Reading Frame (ORF) that encodes a polyprotein containing 3898 amino acids (aa) and a 227-nt 3'-UTR. Researchers compared the sequences of the $\mathrm{HEBZ} / \mathrm{CA} / 2010$ isolate with those of other known CSFV isolates obtained from GenBank. The complete genome of $\mathrm{HEBZ} / \mathrm{CA} / 2010$ shared $83.5-96.3 \%$ identity with that of other reported strains at the nucleotide level. On the basis of the analysis, the isolated HEBZ/CA/2010 was thought to belong to the highly virulent isolate group. This finding is useful for the prevention of CSFV epidemics and the development of a vaccine in China furthermore it contributes to studying the worldwide spread of CSFV.
\end{abstract}

Key words: E0, complete genome, swine fever virus, epidemic strain, nucleotide, polyprotein

\section{INTRODUCTION}

The Classical Swine Fever Virus (CSFV) is the causative agent of Classical Swine Fever (CSF), an economically important disease which has been classified by the Office International Des Epizooties (OIE) (Edwards et al., 2000; Moennig, 2000). The disease is highly contagious and often fatal. The characteristics of CSFV infection are fever and hemorrhages and it can run an acute, chronic or even sub-clinical course.

Together with bovine viral diarrhea virus and border disease virus, CSFV is a member of the genus Pestivirus within the family Flaviviridae (Heinz, 2004) and is epidemic in many countries of Eastern Europe, South-Eastern Asia and Southern and Central America (Paton et al., 2000; Paton and Greiser-Wilke, 2003; Pan et al., 2005; Sabogal et al., 2006). Now a days although, CSFV has been eradicated it is still endemic in some wild boar populations and farm pigs and this increases the risk of an outbreak in farms (Andrew et al., 2000; De Smit et al., 2001).
CSFV is a small enveloped virus that has a single-stranded RNA genome of about $12.3 \mathrm{kbp}$. Its genome consists of 1 Open Reading Frame (ORF) flanked by highly conserved $5^{\prime}$ and $3^{\prime}$-Untranslated Regions (UTRs) (Xiao et al., 2004). The ORF is translated into a polyprotein which is co and post-translationally processed into the individual structural and nonstructural proteins by viral and cellular proteases. C, E0, E1 and E2 are the structural proteins in CSFV. The E0 sequence is one of the most highly mutated regions of the CSFV genome and plays an important role in the reproduction of the virus. The $\mathrm{E} 0$ sequence also plays a major role in virus growth and spread in host cells. Thus, the $E O$ gene can be used to measure the severity of CSFV infection (Meyers and Thiel, 1996).

This study aimed to elucidate the origin and evolution of viruses responsible for the CSF epidemic by using isolates collected from 14 provinces in China during 2010-2011. The strains were detected by Reverse Transcription-Polymerase Chain Reaction (RT-PCR) (Barlic-Maganja and Grom, 2001; Aoki et al., 2002;

Corresponding Author: Jinqing Jiang, College of Animal Science, Henan Institute of Science and Technology, 453003 Xinxiang, China 
Risatti et al., 2003; Hoffmann et al., 2005; Haegeman et al., 2006; Liu et al., 2007). The sequences of the $E O$ gene s were obtained in the same way. By analyzing the $E O$ genes, researchers identified the epidemic strain that spread in China during 2010-2011. Finally, researchers determined the whole sequence of the epidemic strain by using others methods (Li et al., 2006).

\section{MATERIALS AND METHODS}

Clinical samples, cells and viruses: About 854 clinical samples (lungs, livers and kidneys) were collected from clinically ill pigs in the Beijing, Chongqi, Guangxi, Hebei, Henan, Heilongjiang, Hubei, Hunan, Jiangxi, Liaoning, Shandong, Shanxi, Sichuan and Yunnan provinces in China during 2010-2011.

Porcine Kidney cells (PK15) are conserved in the China Animal Health and Epidemiology Center. These cells are cultured in Dulbecco's Modified Eagle Medium (DMEM) containing $10 \%$ fetal bovine serum. Cultures were maintained at $37^{\circ} \mathrm{C}$ in a direct heat $\mathrm{CO}_{2}$ incubator with humidified atmosphere and $5 \% \mathrm{CO}_{2}$.

The clinical samples were grown in PK15 cells to increase viral concentration and to easily extract their RNA. The viruses did not cause any pathological change during incubation in the cells.

RNA extraction, reverse transcription and nest-PCR detection: Viral RNA was obtained by Trizol LS Reagent (Invitrogen) extraction of the PK15 cells that were infected with the respective virus. The RNA was then reversetranscribed using reverse transcriptase XL (AMV) and a ribonuclease inhibitor (TAKARA). To detect the CSFV in the clinical samples, researchers designed the following oligonucleotide primers on the basis of the sequence of the CSFV strain GXWZ02 (AY367767):

\section{- PF1: CGCCACTCAGATTACTTC \\ - PR1: TTCCATAACCAGCCCTTT \\ - PF2: GCATTGCTTATGgTtAG \\ - PR2: AAGTGGGCAGTATGAGG}

Nested-PCR is a method to detect CSFV easily which is used to amplify microscale virus from samples. Nset-PCR is good for reducing the possibility of amplifying other target sites and increasing limited target sensitivity. PF1 and PR 1 are the primers used for the first PCR and PF2 and PR2 are those used for the second PCR. Both PCRs were performed in total volumes of $25 \mathrm{~L}$ with 35 cycles at $94^{\circ} \mathrm{C}$ for $1 \mathrm{~min} 55^{\circ} \mathrm{C}$ for $1 \mathrm{~min}$ and $72^{\circ} \mathrm{C}$ for $1 \mathrm{~min}$ followed by a final extension at $72^{\circ} \mathrm{C}$ for $10 \mathrm{~min}$.
$E O$ gene cloning and phylogenetic analysis: Researchers obtained the EO genes by using the following oligonucleotide primers which were designed on the basis of the sequence of the CSFV strain GXWZ02 (AY367767):

- E01F: AACCACCAGAATCTAGGAAG

- E01R: GTGTTTTTGgGgagGCAAG

- E02R: AAAGCCCTATTGGCATGGG

- E02R: GgTGCAGTTGTtAgtgTAC

The EOgene is the most highly mutated region of the CSFV genome and plays the most important role in the reproduction of the virus and induction of an immune reaction. Analysis of the EO sequence homology with vaccine strain and other strains isolated in China is one way to determine the distribution of CSFVs and to identify the epidemic strain in China.

Whole-genome phylogenetic analysis: Researchers divided the complete genome into 11 fragments and used the primers shown in Table 1 to generate the respective PCR products. The fragments obtained by RT-PCR were cloned into the pGEM-T Easy Vector System (Promega). The respective inserts were sequenced by TAKARA.

The full-length genome sequences of $30 \mathrm{CSFV}$ strains were obtained from GenBank and aligned with the sequences of the strain $\mathrm{HEBZ} / \mathrm{CA} / 2010$ by using the software ClustalX, MegAlign. The phylogenetic tree and nucleotide substitutions were obtained by using the software MEGA4.

Table 1: The synthetic oligonucleotides used for amplification of the complete CSFV genome

\begin{tabular}{lll}
\hline Name & Polarity & Sequence (5'-3') \\
F1 & Sense & GTATACGAGGTTAGTTCATTC \\
R1 & Antisense & CTTCCTAGATTCGGTGGT \\
F2 & Sense & GATAGCCCCAAAAGAGCATG \\
R2 & Antisense & GTACCTATCGCATTGGTTGATG \\
F3 & Sense & CAGATTGGTGGCCGTATGAAAC \\
R3 & Antisense & GCAGCGGCGAGTTGTCTGTTAG \\
F4 & Sense & GCACGAGTGCTTGATTGGCAAC \\
R4 & Antisense & CATCAGCGGACCATTACTTCATT \\
F5 & Sense & CACCATGTTGCCATTGATC \\
R5 & Antisense & TGATGCTGTCACACAGGTG \\
F6 & Sense & CATCAGTTCAGTGGACCATGT \\
R6 & Antisense & CGTCACTATGAAGGGCATCTT \\
F7 & Sense & TGACATCGCAGTCCCCTTACGTGG \\
R7 & Antisense & CTAAAGCCGGTTCAACTATCTTGG \\
F8 & Sense & GCGACTTACACATACAAAAGCTG \\
R8 & Antisense & GTGTAAGCCAGCCAGGTTGTAAC \\
F9 & Sense & GGTTCATTCTCTGCAGAAAT \\
R9 & Antisense & TCAGGCGGTCCAGTCATCATT \\
F10 & Sense & CCTGAGGACCAAACACATGTTG \\
R10 & Antisense & TCAGTGGGTTCCAGGAGTACATC \\
F11 & Sense & ACGGAAGGGGACAAGATGAAAG \\
R11 & Antisense & GGGCCGTTAAATTACCTTAGTC \\
\hline & &
\end{tabular}




\section{RESULTS AND DISCUSSION}

Pathological characteristics of the clinical samples: The representative sick pigs had the following symptoms: rubefaction, blood spots, high fever $\left(40-42^{\circ} \mathrm{C}\right)$, anorexia, asthma, depression, shivering, respiratory tract disorder and diarrhea. To detect pathological changes, autopsies were performed on representative dead pigs (Fig. 1). The autopsies demonstrated severe damage of the organs (lungs, spleen, liver, etc.) e.g., lung edema, frequent blood spots in the kidney and button-like ulcer at the junction of the ileum and cecum.

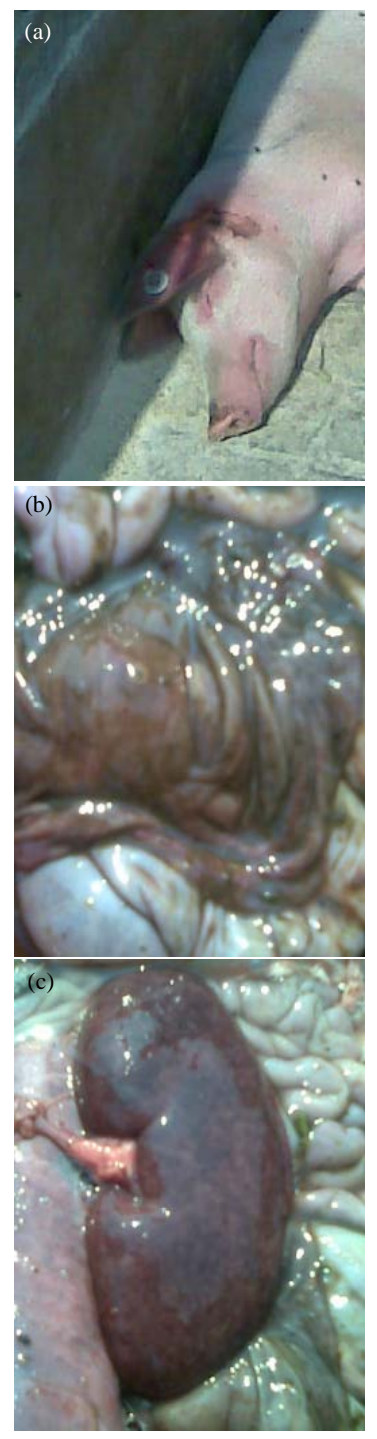

Fig. 1: Symptoms at clinical presentation of a pig with CSFV infection. a) Killed pig with rubefaction; b) Button-like ulcer at the junction of the ileum and cecum and c) Kidney with hemorrhage
RT-PCR identification of clinical samples infected with CSFV: On performing nested RT-PCR, CSFV-infected samples yielded a PCR product of about $500 \mathrm{bp}$. Of the 854 samples 125 were CSFV positive and the infection rate was about $14.6 \%$. The results of representative CSFVpositive samples are shown in Fig. 2.

The 681 bp $E \theta$ gene product obtained by PCR of CSFVpositive samples: On performing nested RT-PCR using specific primers for the $\mathrm{EO}$ sequence, CSFV-positive samples yielded, a PCR product of $681 \mathrm{bp}$ and the results are shown in Fig. 3.

Analysis of the E0 sequences of isolates from 14 provinces in China collected during 2010-2011: In this studying, $40 \mathrm{EO}$ sequences have got 39 sequences of

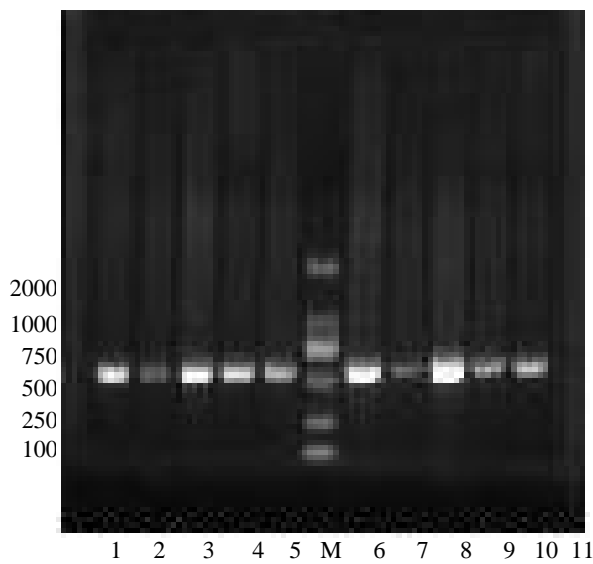

Fig. 2: PCR products of about $500 \mathrm{bp}$ obtained from CSFV-positive samples. Lane M: DL 2000 DNA marker; Lanes 1-9: CSFV-positive samples; Lane 10: Positive control; Lane 11: Negative control

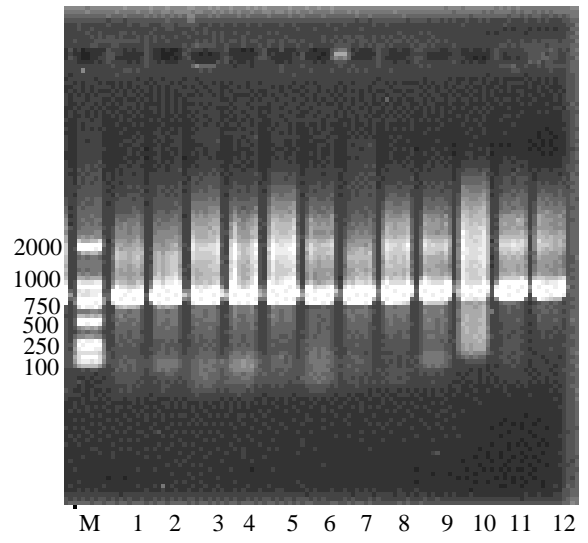

Fig. 3: PCR products (681 bp) of the $E 0$ gene. Lane M: DL 2000 DNA marker; Lane 1: Positive control; Lanes 2-12: CSFV-positive samples 
isolates from 14 provinces and 1 from the CSFV vaccine. Analysis of the EO genes on the basis of sequence similarity and by using a phylogenetic tree (Fig. 4) showed that the strain HEBZ/CA/2010 may be the epidemic one because it has the highest homology with most of the other strains that were isolated in the 14 provinces.

Except for the strain of vaccine most of the EO genes from CSFV strains have high homology (Fig. 4) and they shared 79.4-82.1\% identify with VACCINE at nocleotide level. By sequence analysis, SX1/CA/2010, JX1/CA/2010, $\mathrm{HUB} 1 / \mathrm{CA} / 2010, \mathrm{CQ} 1 / \mathrm{CA} / 2010$ and $\mathrm{YN} 1 / \mathrm{CA} / 2010$ may belong to the same strain of CSFV; the other strains belong to another strain which strains shared $91 \%$ identify at nocleotide level. In other words, there are only two strains of CSFV, one is spread in SX,

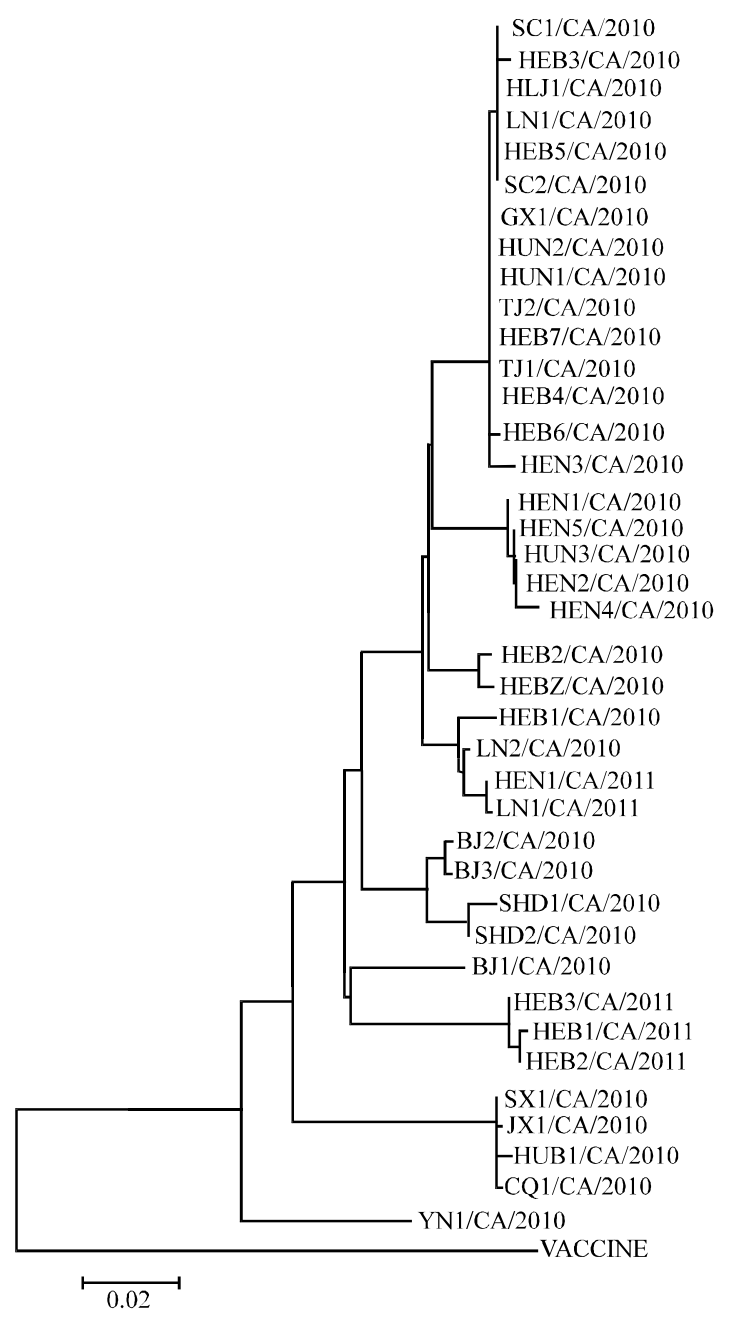

Fig. 4: Analysis of the $E O$ genes with respect to other strains isolated in 14 provinces in China between 2010 and 2011
$\mathrm{JX}, \mathrm{HUB}, \mathrm{CQ}, \mathrm{YN}$ provinces and the other is spread in $\mathrm{SC}$, HEB, HLJ, GX, TJ, HEN, LN, BJ and SHD provinces. Finally, HEBZ/CA/2010 is chosen as the epidemic strain because it belongs to the strain that spread widely in China.

Complete genome sequencing and phylogenetic analysis: The complete genome sequence of the CSFV strain HEBZ was amplified and copied into 11 overlapping DNA fragments by RT-PCR. All DNA fragments were cloned into the pGEM-T Easy Vector system and then sent for sequencing to TAKARA.

The CSFV-positive samples yielded 11 overlapping PCR products (Fig. 5) which consist of the complete genome sequences.

The complete genome sequence containing 12286 nucleotides was submitted to the GenBank (Accession No: GU592790). The coding region starts at nucleotide 372 and ends at nucleotide 12068 which translates into 3898 amino acids and exhibits a genomic organization similar to that of other CSFVs. The 5'-UTR of 371 nucleotides is followed by an ATG translation initiation codon at position 372 and a single ORF of 11697 nucleotides encoding 4 structural and 8 non-structural proteins. The 3'-UTR contains 228 nucleotides.

The complete genome of $\mathrm{HEBZ} / \mathrm{CA} / 2010$ shared $83.5-96.3 \%$ identity with that of other reported strains at the nucleotide level. The epidemic strain HEBZ/CA/2010

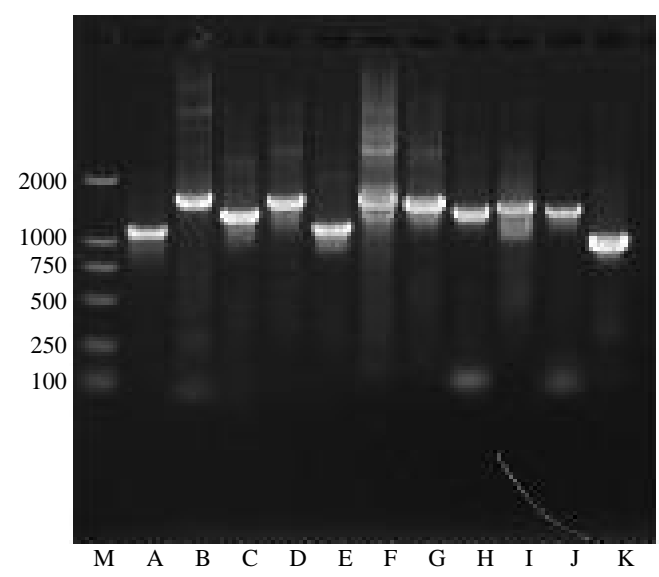

Fig. 5: The PCR results of the complete CSFV genome that was divided into 11 parts. Lane M: DL 2000 DNA marker; Lane A: Part 1, about 1110 bp; Lane B: Part 2, about 1554 bp; Lane C: Part 3, about 1320 bp; Lane D: Part 4, about 1543 bp; Lane E: Part 5, about 1134 bp; Lane F: Part 6, about 1476 bp; Lane G: Part 7, about 1420 bp; Lane H: Part 8, about 1314 bp; Lane I: Part 9, about 1468 bp; Lane J: Part 10, about 1348 bp; Lane K: Part 11, about 1023 bp 


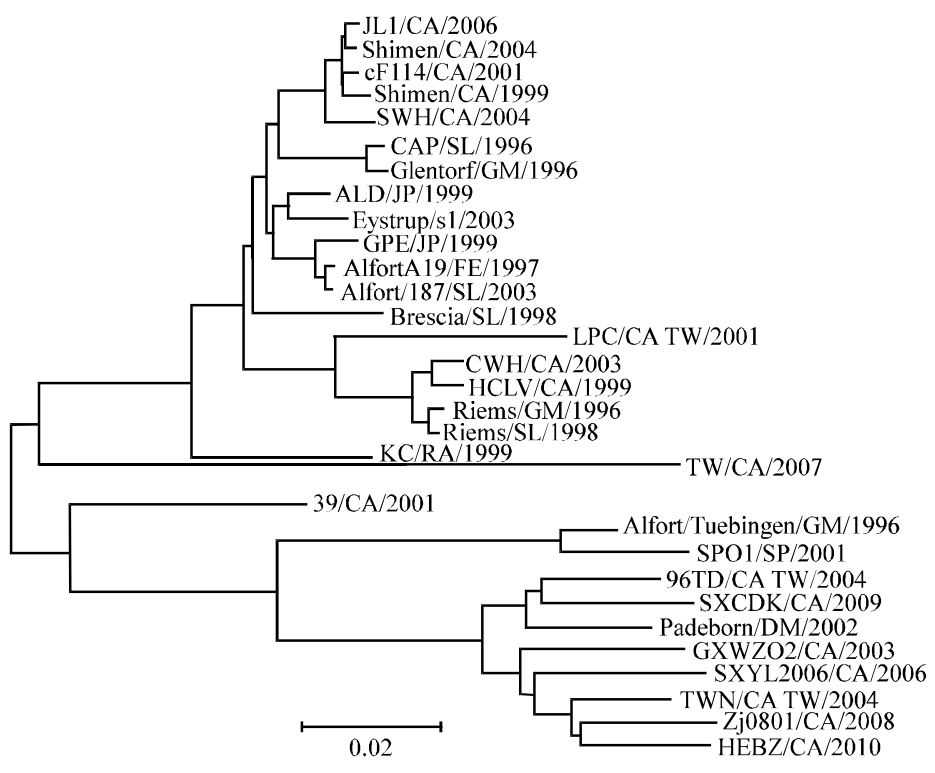

Fig. 6: Analysis of the complete genome with respect to that of other strains reported in GenBank

is closely related to the strain ZJ0801/CA/2008 and they may have similar virulence. The result is shown in Fig. 6. The CSFV strains in the upper part belong to the moderately virulent strains (Fig. 6) and infection may lead to poor reproductive performance and the birth of piglets with neurologic defects such as congenital tremor. Strains in the lower part are highly virulent causing acute symptoms and high mortality and are not susceptible to vaccination. Now a days most of the epidemic strains are highly virulent.

CSF has been recognized as an important swine disease. Recently, CSFV has spread worldwide. It is endemic in swine-cultivating countries causing enormous economic losses each year (Moennig, 2000; Xiao-An et al., 2008). There are several reports about the antigenicity structure and inheritance characteristics of this virus. The purpose of this study was to investigate the CSFVs from 14 major pig-cultivating provinces in China. The CSFV strains in this study were from the Northeastern, Northern, Central, Southwestern and Eastern parts of China which are the most intensive stockbreeding areas. The CSFV strain identified in this study can be considered as the epidemic strain in China. Although, the strain HEBZ/CA/2010 and other strains were isolated from different areas most of them have high homology and similar virulence. In this study by analyzing sequence homology, the complete genomic sequence of the epidemic strain in China could be determined. The epidemic strain HEBZ/CA/2010 has some differences compared to the other strains reported in GenBank and the most related CSFV strain is
ZJ0801/CA/2008. Both the above-mentioned strains belong to the genotype 2.1. In China most of the CSFV strains belong to the $2.1,2.2$ or 1.1 genotype and the CSFV strains with the genotype 2.1 or 2.2 are highly virulent often resulting in CSF outbreaks in pig farms. Because of the immunity provided by vaccination in China, CSF is almost always caused by highly virulent strains.

\section{CONCLUSION}

Epidemiological research and analysis of viral genotypes revealed that an increasing number of new strains invade and spread in China (subgroups 2.1 and 2.2) and replaced the old strains (subgroup 3.4). Because of the difference in viral replication ability and their high activity subgroup 2.1 viruses compete with other kinds of virus strains all the time. Vaccines against CSF target CSFV strains that belong to the subgroup 1.1, e.g., C-strain and Shimen-strain which are moderately virulent strains. On the other hand vaccination may not provide protection against subgroup 2.1 viruses (Ruggli et al., 1996; Widjojoatmodjo et al., 2000).

\section{ACKNOWLEDGEMENTS}

The researchers would like to thank Dr. Jianhua Wang and Lei Zhang for critically reviewing the manuscript. This research was supported by the Laboratory of Animal Epidemiological Surveillance, Animal Health and Epidemiology Center China. 


\section{REFERENCES}

Andrew, M.E., C.J. Morrissy, C. Lenghaus, P.G. Oke and K.W. Sproat et al., 2000. Protection of pigs against classical swine fever with DNA-delivered gp55. Vaccine, 18: 1932-1938.

Aoki, H., T. Shimazaki, C. Takahashi, Y. Sasaki, S. Suzuki and A. Fukusho, 2002. Method for detection of extraneous active bovine viral diarrhoea virus and classical swine fever virus in animal viral vaccines by RT-PCR, which amplify negative-strand viral RNA in infected cells. Biologicals, 30: 27-35.

Barlic-Maganja, D. and J. Grom, 2001. Highly sensitive one-tube RT-PCR and microplate hybridisation assay for the detection and for the discrimination of classical swine fever virus from other pestiviruses. J. Virol. Methods, 95: 101-110.

De Smit, A.J., A. Bouma, E.P. de Kluijver, C. Terpstra and R.J.M. Moormann, 2001. Duration of the protection of an E2 subunit marker vaccine against classical swine fever after a single vaccination. Vet. Microbiol., 78: 307-317.

Edwards, S., A. Fukusho, P.C. Lefevre, A. Lipowski, Z. Pejsak, P. Roehe and J. Westergaard, 2000. Classical swine fever: The global situation. Vet. Microbiol., 73: 103-119.

Haegeman, A., J. Dewulf, R. Vrancken, M. Tignon, S. Ribbens and F. Koenen, 2006. Characterisation of the discrepancy between PCR and virus isolation in relation to classical swine fever virus detection. J. Virol. Methods, 136: 44-50.

Heinz, F.X., 2004. Eighth Report of the International Committee on Taxonomy of Virus. Academic Press, New York, USA.

Hoffmann, B., M. Beer, C. Schelp, H. Schirrmeier and K. Depner, 2005. Validation of a real-time RT-PCR assay for sensitive and specific detection of classical swine fever. J. Virol. Methods, 130: 36-44.

Li, X., Z. Xu, Y. He, Q. Yao and K. Zhang et al., 2006. Genome comparison of a novel classical swine fever virus isolated in China in 2004 with other CSFV strains. Virus Genes, 33: 133-142.

Liu, L., F. Widen, C. Baule and S. Belak, 2007. A one-step, gel-based RT-PCR assay with comparable performance to real-time RT-PCR for detection of classical swine fever virus. J. Virol. Methods, 139: 203-207.
Meyers, G. and H.J. Thiel, 1996. Molecular characterization of pestiviruses. Adv. Virus Res., 47: 53-118.

Moennig, V., 2000. Introduction to classical swine fever: Virus, disease and control policy. Vet. Microbiol., 73: 93-102.

Pan, C.H., M.H. Jong, T.S. Huang, H.F. Liu, S.Y. Lin and S.S. Lai, 2005. Phylogenetic analysis of classical swine fever virus in Taiwan. Arch. Virol., 150: 1101-1119.

Paton, D.J. and I. Greiser-Wilke, 2003. Classical swine fever-an update. Res. Vet. Sci., 75: 169-178.

Paton, D.J., A. McGoldrick, I. Greiser-Wilke, S. Parchariyanon and J.Y. Song et al., 2000. Genetic typing of classical swine fever virus. Vet. Microbiol., 73: $137-157$.

Risatti, G.R., J.D. Callahan, W.M. Nelson and M.V. Borca, 2003. Rapid detection of classical swine fever virus by a portable real-time reverse transcriptase PCR assay. J. Clin. Microbiol., 41: 500-505.

Ruggli, N., J.D. Tratschin, C. Mittelholzer and M.A. Hofmann, 1996. Nucleotide sequence of classical swine fever virus strain Alfort/187 and transcription of infectious RNA from stably cloned full-length cDNA. J. Virol., 70: 3478-3487.

Sabogal, Z.Y., J.D. Mogollon, M.A. Rincon and A. Clavijo, 2006. Phylogenetic analysis of recent isolates of classical swine fever virus from Colombia. Virus. Res., 115: 99-103.

Widjojoatmodjo, M.N., H.G.P. van Gennip, A. Bouma, P.A. van Rijn and R.J.M. Moormann, 2000. Classical swine fever virus $\mathrm{e}^{\mathrm{rns}}$ deletion mutants: Transcomplementation and potential use as nontransmissible, modified, live-attenuated marker vaccines. J. Virol., 74: 2973-2980.

Xiao, M., J. Chen, Y. Wang, Y. Zhen, W. Lu, J. Chen and B. Li, 2004. Sequence, necessary for initiating RNA synthesis, in the 3'-noncoding region of the classical swine fever virus genome. Mol. Biol., 38: 343-351.

Xiao-An, C., H. Hui, L.G. Zhen, Q.C. Qing and Z.J. Zhang, 2008. Differential diagnosis of antibody to classical swine fever virus field strain by ELISA with recombinant $\mathrm{E} 2$ proteins of various group CSFV. J. Anim. Vet. Adv., 7: 972-980. 